\title{
Critical analysis of existing business process reengineering models: towards the development of a comprehensive integrated model
}

Bassam Hussein, Hassan Bazzi, Ayman Dayekh, Walid Hassan

Lebanese International University

\begin{abstract}
Over the past two decades, business process reengineering (BPR) has been one of the most popular approaches to improving the efficiency and effectiveness of an organisation. However, a review of the relevant literature reveals that available BPR models that have been widely in use have some serious limitations and fail to take into consideration the human factor and change management. The theoretical gap identified in academic research is also reflected in industrial practice. This article analyses existing BPR models in order to set the stage for the development of a comprehensive integrated model to address their theoretical deficiencies. The article will provide an overview of the main issues, limitations and challenges of existing BPR models. The limitations will then be itemised, described and analysed to provide evidence for the need of a more systematic model that would help organisations to successfully carry out BPR initiatives and projects.
\end{abstract}

Keywords: Business process reengineering (BPR), BPR models, organisational change, change management, human factors, human resources management

\section{Introduction}

BPR has become a widely used approach since the early 1990s, yielding potential benefits such as increasing productivity through reduced process time and cost, improved quality and greater customer satisfaction (Cao, et al. 2001). Kontio (2007) provides an operational definition of BPR as an approach where processes are developed to maximise an organisation's potential. Many industries across the globe have carried out BPR initiatives that span over a wide range of sectors such as services, information and communications technology (ICT), healthcare, manufacturing, production, construction, education, public service, government. The promise of substantial financial gain has motivated numerous firms across a wide range of industries to adopt major process change initiatives, with some achieving significant benefits (Ramirez, et al. 2012).

Several strategies and models have been developed for the implementation of BPR (Chi-Kuang \& Cheng-Ho 2008). However, most of the business organisations that have carried out BPR initiatives have followed a traditional approach using conventional linear life cycle models. Unfortunately, these models fail to serve the objectives of BPR. Most of the existing BPR models were inspired by early and primitive software development and engineering approaches that have always been criticised for their linear and static nature. In such approaches, the reengineering effort is broken down into stages where the output of one stage serves as the input of the next. Both the diagnosing and transforming phases must be carried out before any implementation is attempted. According to Petrozzo and Stepper (1994), the waterfall method is fundamentally flawed. Like any other process, where many people are involved and those people are functionally aligned, the traditional waterfall life cycle model is slow, error-prone and inflexible. Thus, this approach is not suited to the current competitive operating environments. In a reengineering project, the need to abandon the traditional approach is particularly pronounced. 
Where there is an incomplete knowledge of the details of the redesign, it is necessary to test the concepts while they are still in their infancy (Petrozzo \& Stepper 1994).

Accurate and complete representation and analysis of business processes are crucial to the success of BPR. The objectives of using a BPR model may be classified as communication, analysis and control. Regarding communication, facilitating the understanding of business processes may be the primary objective of using a BPR model (Hussein 2008). Process designers need to describe existing and improved processes, agree upon a common representation and share their knowledge of business processes with other stakeholders. Simplicity and clarity may be the most desired features of a BPR model for the purpose of communication. According to Cao et al. (2001), BPR failure can frequently be traced to ineffective communication. On the analysis front, analysing and improving existing processes may be another primary cause for the use of BPR models. In order to identify the best process, process engineers and designers need to generate alternative representations, simulate process behavior and measure process performance. In addition to communication and analysis, a BPR model could also be used for managing and monitoring business processes. Given many interrelated processes within a firm, process engineers need to oversee process operations, manage process relationships and audit process performance (Luo \& Tung 1999). Hussein (2008) provides an extensive list of key success factors distilled from various articles and empirical research on BPR. He argues that the most relevant BPR success factors include, but are not limited to:

- $\quad$ organisation-wide commitment,

- BPR team composition,

- business needs analysis,

- adequate IT infrastructure,

- effective change management, and

- ongoing continuous improvement.

\section{Conventional BPR models analysis}

A plethora of BPR models have appeared in the literature during recent years; however, most of them have serious limitations mainly due to the need for a multidisciplinary approach (Valiris \& Glaykas 1999). Establishing a disciplined model for BPR and using a sound approach are prerequisites to BPR success. A BPR model should be designed or selected creatively to satisfy the current needs of the organisation and support the life phases of a BPR project (Chatha, et al. 2007). Source: Carr and Johansson (1995)

, compiled by Carr and Johansson (1995), presents briefly a set of elements of a successful BPR model. BPR methodologies can be categorised in different ways (Eftekhari \& Akhavan 2013). BPR literature suggests that existing process reengineering models may be classified into inhouse and outside models. The advantages of an in-house model are that it comes from a familiar cultural base and often presents ideas in a way employees are familiar with. The disadvantage, of course, is that too much familiarity could allow people to slide through a reengineering effort without ever trying to shatter the paradigms within which they currently operate. On the other hand, the advantages of using an outside BPR model is that it is based on a breadth of experience in other organisations. The disadvantage is that, if it is not presented well or constantly revised to 
take into account the rapid changes in business, it can appear like a cookie-cutter approach (Carr \& Johansson 1995).

Table 1. Elements of a successful BPR model

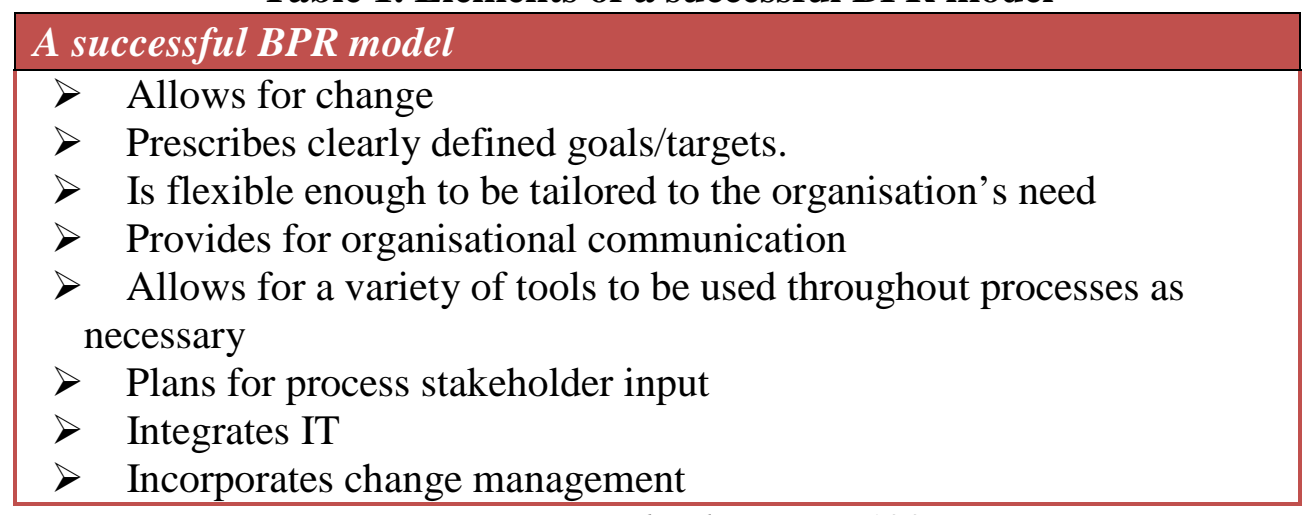

Source: Carr and Johansson (1995)

Since the application of BPR concepts can have different forms, its methodologies are different (Eftekhari \& Akhavan 2013). The primary functions of a BPR model are to determine the order of the stages involved in reengineering projects, their evolution, and to establish the transition criteria for piloting the progress from one stage to the next. This includes the completion criteria and the choice criteria for the current stage, and the entrance criteria for the next stage. BPR models provide guidance on the order in which a project should carry out its major tasks. Many BPR projects have failed because they pursued their various reengineering and evolution phases in the wrong order. This failure is evident in some of the existing BPR models where the stages are not well defined and their order is not presented appropriately.

The development of BPR models and frameworks has been greatly influenced by the evolution of BPR itself. Many BPR proponents argue that there are two approaches to BPR (Eftekhari \& Akhavan 2013). The first one involves looking at existing processes and attempting to make improvements to them by redesigning them. In this approach, existing processes are critically examined and worked through to find new processes that produce stepped improvements in outcome. This approach is closer to the streamlining approaches, but its critics argue that the aim of BPR is to have major change implemented rather than small incremental changes (Hussein 2008). The second approach relies on the desired outcome required and investigates the best process design for achieving pre-identified outcomes. In this approach, frequently referred to as the 'clean slate' approach, the perception is that looking too closely at existing processes may unduly influence how a new and better set of processes may be designed. This approach requires a fundamental rethink of how a product or a service is delivered and a design of brand new processes on a 'clean slate'. Davenport and Stoddard (1994) argue that a blank sheet of paper used in design usually requires a blank cheque for implementation. Many companies deploy a combination of both approaches which they refer to as the hybrid BPR technique. Both approaches look at those processes that add value for the customer. They also require management commitment, belief, time and action. Moreover, they need to take account of the people and technology that enable the process (Green 2004). However, one of the main problems of technology is the increase of expenditures related to it as the biggest enabler of BPR (Jafari, et al. 2012). A more affordable approach for most companies is to use a clean slate design, which entails a detailed vision for a process without any concern for the existing environment. 
However, the implementation is done over several phased projects (Malhorta 1998). A summary of the major differences between the two approaches is captured in Table 2.

Table 2: Differences between the clean slate and detailed process analysis approaches

\begin{tabular}{|l|l|l|}
\cline { 2 - 3 } \multicolumn{1}{l|}{} & Clean Slate Approach & Detailed Process Analysis \\
\hline Start & Clean slate & Existing processes \\
\hline Duration & Long & Short \\
\hline Enabler & Broad and cross-functional & $\begin{array}{l}\text { Narrow within silo } \\
\text { functions }\end{array}$ \\
\hline Engagement & ICT & Statistical control \\
\hline Risk Level & Top-down & Bottom-up \\
\hline Change Type & High & Moderate \\
\hline Change Frequency & Once & Cultural \\
\hline Change Level & Revolutionary & Once and/or continuous \\
\hline
\end{tabular}

There are two aspects that are often distinguished in business process modeling: the static organisation structure, and the dynamic behaviour. This distinction has led to the emergence of static and dynamic BPR models. Dynamic models are always more complex than static ones, but the information obtained is invariably richer (Barber, et al. 2003). Most of the conventional BPR models, including those presented earlier, are linear and static in nature. They follow a sequential reengineering approach where a given phase is not initiated until the previous one is completed. A major drawback of most of the traditional sequential process modelling approaches is that they attempt to represent a dynamic process with a two-dimensional static image (Barber, et al. 2003). The disadvantage of using static models is that they do not facilitate the outcome of a changed process to be predicted. Furthermore, the physical aspects of the process, such as resources and technology, cannot be modelled. However, despite the rigidity of static modelling, over 80 per cent of business process change deployments adopt this technique (Gladwin \& Tumay 1994). Static models are not suitable for BPR initiatives because business processes evolve and grow over time. Even though the limitations of static models have been known for some time and recognised by systems analysts and designers for over a decade, it is only recently that dynamic modelling has been considered an essential component of process modelling (Barber, et al. 2003).

BPR is concerned with fundamentally rethinking and redesigning business processes to obtain dramatic and sustaining improvements in quality, cost, lead times, outcomes, flexibility and innovation (Hammer \& Champy 1993). A serious problem with conventional BPR models is the long cycle time for delivering results, with even the most aggressive projects typically requiring 
six months to reach new designs and over a year to implement (Davenport 1995). Apart from offering an inadequate ontology for modelling, traditional approaches can also be criticised for providing little for the analysis of organisational structures. It is common in many conventional BPR models that features of the business process are represented early in the reengineering effort by abstract objects and operations. This implies that it is necessary to preconceive the possible future states for the model and the proposed process. For example, using object-oriented models, the objects, containing attributes and methods, need to be defined and carefully planned in advance before application to the process. These attributes form the data for manipulation in the model and such data will determine the complete operational domain of the process and all possible interactions with it. Such a process can be modified and improved, but it remains an inherent feature of the paradigm that the originally reengineered process forms and becomes a boundary within which the future state and interaction must be preconceived (Chen 2001).

One of the main benefits that BPR brings to the organisation is the focus on core business processes. An organisation decides to engage in BPR because it is seeking to improve its efficiency (Rao, et al. 2012). According to many in the field, reengineering should focus on processes and not be limited to thinking about the organisations. After all, the organisation is only as effective as its processes (Hammer \& Champy 1993). Unfortunately, most businesses are not process oriented; they are focused on tasks, jobs, people and structures, but not on processes (Browne \& O’Sullivan 1995). Due to the rapid growth of interest in BPR, a large number of existing models have been individually upgraded or combined in order to fall under the BPR umbrella (Valiris \& Glaykas 1999). These models; however, do not fully recognise that the concept of business processes is the basic underlying idea of BPR. These processes are characterised by a number of attributes. Based on the most popular definitions of BPR, Simon (2003) compiled the following list of characteristics for a business process:

1. Definability: It must have clearly defined boundaries, input and output.

2. Order: It must consist of activities that are ordered according to their position in time and space.

3. Customer: There must be a recipient of the process's outcome, i.e., a customer.

4. Value-adding: The transformation which is taking place within the process must add value to the recipient either upstream or downstream.

5. Embeddedness: A process cannot exist in itself; it must be embedded in an organisational structure.

6. Cross-functionality: A process regularly can, but not necessarily must, span several functions.

Increasingly, researchers and practitioners are describing business organisations in terms of processes rather than functional hierarchies. An organisation may be viewed as a web of interrelated processes that are designed to achieve certain organisational goals (Luo \& Tung 1999). Based on this view, the reengineering process using linear models raises some problems. Linear models define the requirements in a way from which it may be easy for process engineers to construct specifications. However, processes developed in this way may be resistant to change and extension. The reason is that such methods focus on the 'processing' rather than the 'concepts' of the process (Sully 1993). Sully (1993) points out that processing of a business process is the most volatile aspect of a process, which may change rapidly over time. Other potential problems may occur from the transformation between requirements specification to 
implementation. As Morris et al. (1996) observe, the weakness of all structured methods is that the effort of analysis may be left behind, just when it has reached the peak of its development, and when the development is going into the implementation stage. That is, a new structure will be defined in implementation, which tries to map the products of earlier analysis to some executable functions. Error is likely to occur during this transformation and it will become worse if such error occurs at later stages.

In BPR implementation, it is important that a systematic and structured model is followed, integration is established with other ongoing process improvement initiatives, and a businesscentred performance measurement scheme is developed and adopted (Al-Mashari 2003). In addition, a BPR program is effectively a change management program as well, and its implementation often leads to fundamental change within an organisation's structure, culture and management process (Al-Mashari \& Zairi 2000). BPR failure, however, can frequently be traced to ineffective management of organisational resistance to change or the failure to create the new organisational culture and structure needed to support it. Cao et al. (2001) suggest a classification of four types of organisational change, presented in Table 3. Unfortunately, most of the existing BPR models pay little attention to the human factors and change management ignoring that organisations are made up of actors who perform certain roles to achieve their goals through a network of relationships (Rao, et al. 2012). It has been argued that BPR is the technique that completely forgot people and ignored their impact on organisations. On 26 November 1996, The Wall Street Journal reported that Michael Hammer and other leaders of the reengineering movement forgot about people. Hammer was quoted as saying 'I wasn't smart enough about that ... and was insufficiently appreciative of the human element' (Gore 1999). The human factor plays a critical role in the long-term success and productivity of any business organisation (Campbell \& Kleiner 1997). To survive in the turbulent contemporary environment, business organisations need to adapt to external conditions through change management (Chi-Kuang \& Cheng-Ho 2008).

Table 3: Four types of organisational change

\begin{tabular}{|l|l|}
\hline Change Type & Description \\
\hline Processual & $\begin{array}{l}\text { Changes in organisational process and controls over } \\
\text { process }\end{array}$ \\
\hline Structural & $\begin{array}{l}\text { Changes in organisational functions, their organisation, } \\
\text { coordination, and control. Involves a consideration of } \\
\text { horizontal and vertical structures, the decision systems, } \\
\text { and human resources management. }\end{array}$ \\
\hline Cultural & $\begin{array}{l}\text { Changes in values, beliefs and human behavior in terms } \\
\text { of relationships to social rules and practices. }\end{array}$ \\
\hline Political & $\begin{array}{l}\text { Changes in power distribution and the way organisational } \\
\text { issues are influenced. }\end{array}$ \\
\hline
\end{tabular}

Source: Cao et al. (2001)

\section{Limitations of existing BPR models}

Many existing BPR models are limited in the sense that they only consider a single process separately and allow improvements to that one process, but do not consider the effect of the changes on other processes within the business. It could be that improvements to the studied 
process may have a detrimental impact on the business as a whole. To be absolutely sure that BPR benefits the business, the entire business should be modelled and evaluated. It follows that a holistic model of the entire business should be the ultimate objective (Barber, et al. 2003). In any BPR project, it is important to gain an overall understanding of how the key processes in an enterprise relate to one another before focusing on one or a few processes to reengineer (Browne \& O’Sullivan 1995).

A review of the literature on existing BPR models reveals that these models exhibit many limitations. These limitations may be summarised and classified according to Cao et al.’s (2001) four types of organisational change:

1. Most of the existing BPR models are based on BPR practitioners' experience, with little attention to the analysis of the business organisation's environment or vice versa (Hussein 2008). Change type: cultural.

2. Most of the existing BPR models are founded on how the business processes should change and how organisations should adapt to change rather than on the evaluation of current practices and on the implementation of successful practical experiences (Rao, et al. 2012). Change type: processual and structural.

3. There is a big division in the BPR literature between models that concentrate either on process improvement or process innovation. The main difference is on the way organisational change is understood. In the former case, change is performed in an incremental fashion, whereas in the latter, in a radical way. However, in many cases a combination of the two approaches has yielded the most impressive results (Valiris \& Glykas 1999). Change type: processual.

4. The majority of the existing BPR models are general frameworks attempting to cover the needs of a wide range of business organisations. However, it is very difficult to produce a standard BPR recipe of success for every business sector and under all circumstances (Eftekhari \& Akhavan 2013). Change type: processual and political.

5. Most existing process models are deficient in that they cannot deal with partial descriptions of world states. They only describe processes operationally, i.e., in terms of operations that produce new output states given completely specified input states (Hussein 2008). Change type: processual and structural.

6. There is a need for an integrated, holistic and individualistic view of the organisation. Most existing BPR models concentrate on organisational processes without paying any attention to the roles and responsibilities of the employees who carry out the activities that compose these processes (Valiris \& Glykas 1999). Change type: processual and structural.

7. Most of the current BPR models are developed with the intention of targeting reengineering specialists rather than the team that would be carrying out the BPR initiative in the organisation (Ozcelik 2010). Change type: structural.

8. Some models fail to recognise the importance of the diagnosis phase at the beginning of the reengineering project, where the BPR team evaluates and documents current processes, uncovers bottlenecks and establishes baselines and benchmarks for gauging future improvements (Eftekhari \& Akhavan 2013). Change type: processual. 
9. Most of the process reengineering models stop at the implementation phase and consequently seem to be quite static. The exclusion of evaluation and the notion of continuous improvement seem to be inconsistent with the increasing pressures of an ever-changing world and from a highly competitive environment (Vakola \& Rezgui 2000). Change type: processual.

10. Existing BPR models are not dynamic in nature and use a more black-and-white approach (Hussein 2008). For instance, in some models generic management and the use of information technology are the main objectives whereas in others cost is the central issue. Change type: processual and structural.

11. A major drawback of some BPR models is that they are not cost-effective. This factor is crucial for the successful implementation of the reengineering processes because the companies have to survive in a highly competitive market. Observations from the literature indicate that, although the theory behind the development of certain models is well defined, their application is not feasible, due either to cost and time limits or to lack of information of potential savings (Vakola \& Rezgui 2000). Change type: processual.

12. There is inadequate support for storage and access of gathered information during and after the redesign process, especially for non-participants in the redesign exercise (Valiris \& Glykas 1999). Change type: processual.

13. Business modelling is performed using either inadequate descriptive notations from management accounting or poor graphical notations that were created for software development and do not take into account organisational issues (Valiris \& Glykas 1999). It must be noted that some models include some soft elements in their approach; however, they focus more on harder and more quantifiable elements of the organisation. Change type: structural.

14. There is a lack of integrated tool sets that allow modelling and analysis of the business environment. Most of the existing tools for modelling come from the area of software development and usually concentrate on conceptual business modelling (Valiris \& Glykas 1999). Presently, there is a shortage of business analysis tools that are integrated with BPR models. Change type: processual.

15. When initiating the BPR project, the business analysis is done in a subjective rather than an objective manner (Hussein 2008). Change type: cultural.

16. There is no formal underpinning to ensure consistency across models. When graphical notations are used in business modelling and redesign, there is no means of verifying the logical consistency of the resulting models (Valiris \& Glykas 1999). This creates a feeling of insecurity to the business process re-designers in the sense that their work might be undermined by the organisation's cynics. Change type: political.

17. Most of the available and proposed BPR models are intended for application in business organisations in Western countries. This is due to the fact that the BPR philosophy is more suitable in such an environment. However, BPR should be applied in a multiple organisational structures and in different cultures and countries (Hussein 2008). Change type: structural and cultural. 


\section{Conclusion}

Over the past decade, several BPR models have surfaced to guide business organisations through their process reengineering initiatives and efforts. Like the beginnings of many other disciplines, the initial BPR models were simplistic in nature since BPR was, and still is, a relatively new discipline that has not yet matured. These models suffer from serious limitations and problems due to the fact that they follow a traditional approach to carry out BPR without having the essential elements to address the changing environment of today's business organisations. One of the main objectives of any given model is to provide solutions to frequently occurring problems and to formulate a roadmap for achieving this. Therefore, organisations would prefer to have a BPR model that will lead them to successful process reengineering rather than adding a new liability to their business. Considering that there are sufficient problem situations where more than one type of organisational change might surface, the general recommendation is that BPR should be integrated with a holistic perspective using multiple methods to address the dynamic interactions between different types of organisational change (Cao, et al. 2001).

In this article, an overview of the main issues related to existing BPR models was presented. Those issues were then expanded and further analysed to cover a more in-depth look at the problems of existing conventional BPR models and the traditional approach they follow. It was found that most of these models were developed for other purposes in the first place and were later relabelled to fall under the BPR umbrella. Most of the existing BPR models appear to have serious limitations. These limitations were then summarised and discussed to introduce the need for a more systematic model that would help organisations to successfully perform process reengineering.

The advent of BPR remains a subject of great interest and yet of great controversy. There are interesting avenues for future research on this area. The work presented in this paper sets the stage for the development of a comprehensive efficient BPR model that takes into consideration the limitations presented and proposes mechanisms to address them. Arguably, this research represents a small part of an ongoing investigation into an area in which relatively little practical research has been carried out. The work done here takes a fairly high-level view of the subject and there is much in the way of detailed investigation that could usefully be carried out to further explore this area. More models should be developed to determine where the breakdowns occur in BPR implementation, why they occur and how they can be prevented. Moreover, the issue of measurements should be addressed as very few specific objective measures for BPR failure and success have been proposed.

\section{References}

Al-Mashari, M. 2003, A process change-oriented model for ERP application, International Journal of HumanComputer Interaction, vol. 16, no. 1, 39-55.

Al-Mashari, M., Irani, Z. \& Zairi, M. 2001, Business process reengineering: a survey of international experience, Business Process Management Journal, vol. 7, no. 5, 437-455.

Al-Mashari, M. \& Zairi, M. 2000, Revisiting BPR: a holistic review of practice and development, Business Process Management Journal, vol. 6, no. 1, 10-42.

Barber, K.D., Dewhurst, F.W., Burns, R.L.D.H. \& Rogers, J.B.B. 2003, Business process modeling and simulation for manufacturing management: a practical way forward, Business Process Management Journal, vol. 9, no. 4, 527-542.

Browne, J. \& O’Sullivan, D. 1995, Reengineering the Rnterprise: Proceedings of the IFIP TC5/WG5.7 Working Conference on Reengineering the Enterprise,, Chapman and Hall, London, 131-139. 
Campbell, S. \& Kleiner, B. 1997, New developments in reengineering organizations, Work Study, vol. 46, no. 3, 99103.

Cao, G., Clarke, S. \& Lehaney, B. 2001, A critique of BPR from a holistic perspective, Business Process Management Journal, vol. 7, no. 4, 332-339.

Carr, K.D. \& Johansson, H.J. 1995, Best Practices in Reengineering: What Works and What Doesn't in the Reengineering Process, McGraw-Hill Book Company, New York.

Chatha, K.A., Ajaefobi, J.O. \& Weston, R.H. 2007, Enriched multi-process modelling in support of the life cycle engineering of business processes, International Journal of Production Research, vol. 45, no. 1, 103-141.

Chen, Y.C. 2001, Empirical modeling for Participative Business Process Reengineering, PhD thesis, Department of Computer Science, University of Warwick, Coventry, UK, http://www.dcs.warwick.ac.uk/research/modeling/papers/theses/cheny/index.html (accessed Apr 2013).

Chi-Kuang, C. \& Cheng-Ho, T. 2008, Developing a process re-engineering-oriented organizational change exploratory simulation system (PROCESS), International Journal of Production Research, vol. 46, no. 16, 4463-4482.

Davenport, T. 1995, Business process reengineering: where it's been, where it's going, In Business Process Change: Concepts, Methods \& Technologies, Idea Publishing, Harrisburg, 1-13.

Davenport, T. \& Stoddard, D.B. 1994, Reengineering: business change of mythic proportions, MIS Quarterly, vol. 18, no. 2, 121-126.

Eftekhari, N. \& Akhavan, P. 2013, Developing a comprehensive methodology for BPR projects by employing IT tools, Business Process Management Journal, vol. 19, no. 2, 4-29.

Gladwin, B. \& Tumay, K. 1994, Modeling business processes with simulation tools, Proceedings of the 1994 Winter Simulation Conference, 114-121.

Gore, E.W. Jr. 1999, Organizational culture, TQM, and business process reengineering, Team Performance Management: An International Journal, vol. 5, no. 5, 164-170.

Green, D. 2004, Kaizen JIT and TQM compared and contrasted with business process reengineering, http://www.cs.tcd.ie/dgreen2/BPR.doc (accessed Apr 2013).

Hammer, M. \& Champy, J. 1993, Reengineering the Corporation - A Manifesto for Business Revolution. New York: Harper.

Hussein, B. 2008, PRISM: Process Re-engineering Integrated Spiral Model, VDM Verlag, Berlin.

Jafari, M., Bastani, P., Ibrahimipour, H. \& Dehnavieh, R. 2012, Attitude of health information system managers and officials of the hospitals regarding the role of information technology in reengineering the business procedures: a qualitative study, Healthmed, vol. 6, no. 1, 208-215.

Kontio, J. 2007, Business process re-engineering: a case study at Turku University of Applied Sciences, Proceedings of European and Mediterranean Conference on Information Systems 2007 (EMCIS2007), 2426.

Luo, W. \& Tung, Y.A. 1999, A framework for selecting business process modeling methods, Journal of Industrial Management \& Data Systems, vol. 99, no. 7, 312-319.

Malhorta, Y. 1998, Business process redesign: an overview, IEEE Engineering Management Review, vol. 26, no. 3. http://www.brint.com/papers/enterarch.htm (accessed Apr 2013).

Morris, D., Evans, G., Green, P. \& Theaker, C. 1996, Object Oriented Computer Systems Engineering, SpringerVerlag, London.

Ozcelik Y. 2010, Do business process reengineering projects payoff? Evidence from the United States, International Journal of Project Management, vol. 28, 7-13.

Petrozzo, D. \& Stepper, J. 1994, Successful Reengineering, Van Nostrand Reinhold, New York, 144-157.

Rao, L., Mansingh, G. \& Osei-Bryson, K. 2012, Building ontology based knowledge maps to assist business process re-engineering, Decision Support Systems, vol. 52, 577-589.

Ramirez, R., Melville N. \& Lawler, E. 2012, Information technology infrastructure, organizational process redesign, and business value: an empirical analysis. Decision Support Systems, vol. 49, 417-429.

Simon, K. 2003, BPR in the Pharmaceutical Industry, PhD thesis, Department of Informatics, School of Economics and Commercial Law, Göteborg University. http://www.handels.gu.se/epc/archive (accessed Apr 2013).

Sully, P. 1993, Modeling the World with Objects, Prentice Hall, Englewood Cliffs.

Vakola, M. \& Rezgui, Y. 2000, Critique of existing process re-engineering methodologies: the development and implementation of a new methodology, Business Process Management Journal, vol. 6, no. 3, 238-250.

Valiris, G. \& Glykas, M. 1999, Critical review of existing BPR methodologies: the need for a holistic approach, Business Process Management Journal, vol. 5, no. 1, 65-86. 


\section{About the authors \\ Bassam Hussein, BEng (Computer Engineering), MBA (Technology Management), PhD (Engineering}

Management), is an Assistant Professor in the School of Engineering at the Lebanese International University (LIU). Bassam has worked for many international telecommunication corporations and educational institutions. He has led multimillion-dollar software development projects and rolled out many products that are still widely in use across the globe. He has supervised several academic projects, and has participated in many conferences, symposiums and workshops.

Email: bassam.hussein@liu.edu.lb

Hassan Bazzi, MS (High Frequency Electronics and Optoelectronics), PhD (Limoges), is the Chair and an Associate Professor in the Department of Electrical and Electronic Engineering at LIU. His research interests include the design and realisation of LNA on SiGe BiCMOS technology, testing and design techniques using balanced differential structure using this technology, and development and design of differential active filter at microwave frequencies using this technology.

Ayman Dayekh, BSc (Computer Science), MBA, is the Chief Technology Officer at LIU. He has led several IT projects in education and has been involved in designing and managing the execution of data centres and large networks. His current interests are in instructional design and technologies as well as online and e-learning.

Walid Hassan, Dip (Biomedical Engineering), PhD (Industrial Imaging), DEA. He is an active researcher in tomographic reconstruction for emission and transmission data and has contributed to a guide for the good practice of biomedical maintenance and management in hospitals. Walid is a cofounder and Secretary of the Lebanese Society for Biomedical Engineering. 